\title{
Aspectos epidemiológicos de puérperas e neonatos de um Serviço de Parto Normal
}

\author{
Epidemiological Aspects of Puerperal Women and \\ Newborns in a Normal Birth Service
}

RESUMo - Introdução: Importante compreender o perfil epidemiológico de puérperas e recém-nascidos, pois é possível obter dados que contribuem para o planejamento de estratégias e de ações para uma assistência qualificada a essa clientela. Objetivo: Conhecer os aspectos epidemiológicos das puérperas e dos neonatos atendidos em um Serviço de Parto Normal de um município da Bahia. Métodos: Foram avaliadas 276 puérperas e 276 recém-nascidos (RN) atendidos no Serviço de Parto Normal de um hospital do interior da Bahia/Brasil. A coleta dos dados ocorreu por meio de entrevista à puérpera $\mathrm{e}$ da análise do cartão de gestante e dos prontuários das puérperas e dos recém-nascidos. Resultados: A maior parte das puérperas possuía faixa etária de 17 a 26 anos (60,5\%), eram casadas ou mantinham uma união estável $(86,6 \%)$, primigestas $(43,5 \%), 5,0 \%$ foram submetidas à episiotomia e $94,6 \%$ dos partos foram assistidos por enfermeiro obstétrico. A maioria dos recém-nascidos era a termo $(88,4 \%)$, possuía perímetro cefálico entre 31,5 e $37 \mathrm{~cm}(91,3 \%)$ e teve índice de Apgar maior que 7 e 8 no primeiro $(96,0 \%)$ e no quinto $(98,5 \%) \mathrm{mi}$ nuto de vida, respectivamente e $4,8 \%$ nasceram com baixo peso. Conclusão: Os achados do estudo podem contribuir para o corpo de evidências em questão e elucidam que as puérperas, em sua maioria convivem com companheiro, têm mais de 6 anos de estudo, realizaram mais de 6 consultas de pré-natal, fizeram uso de sulfato ferroso na gestação e não tinham comorbidades.

Palavras-chave: Gestante;Recém-nascido;Epidemiologia; Parto normal.

ABstraCT - Introduction: It is important to understand the epidemiological profile of mothers and newborns, as it is possible to obtain data that contribute to the planning of strategies and actions for qualified assistance to this clientele. Objective: To know the epidemiological aspects of puerperal women and newborns treated at a Normal Childbirth Service in a municipality in Bahia. Methods: Study with 276 mothers and 274 newborns attended at the Normal Delivery Service of a hospital in the interior of Bahia/Brasil. Data collection occurred through the analysis of the pregnant woman's card, the medical records of the mothers and newborns and other sector records. Results: Most puerperal women were aged between 17 and 26 years old $(60,5 \%)$, were married or maintained a stable relationship $(86,6 \%)$, primiparous $(43,5 \%), 5,0 \%$ were submitted to episiotomy and $94,6 \%$ of births were attended by an obstetric nurse. Most newborns were term $(88,4 \%)$, had a head circumference between 31,5 and $37 \mathrm{~cm}(91,3 \%)$ and had an Apgar score greater than 7 and 8 in the first $(96,0 \%)$ and in the fifth $(98,5 \%)$ minute of life, respectively and $4,8 \%$ were born with low weight. Conclusion: The findings of the study may contribute to the body of evidence in question and elucidate that the mothers, mostly living with a partner, have more than 6 ye-

Silas Santos Carvalho

ISanta Casa da Misericórdia de Ruy

Barbosa, Ruy Barbosa/BA - Brasil ars of education, attended more than 6 prenatal consultations, used ferrous sulfate in the pregnancy and had no comorbidities.

Keywords: Pregnant woman; Infant;Newborn;Epidemiology; Natural childbirth. 


\section{INTRODUÇÃO}

A assistência obstétrica e neonatal deve ocorrer com qualidade e de forma humanizada desde o momento em que a gestante é acolhida na maternidade até o puerpério imediato. ${ }^{1}$ Para que isso ocorra, alguns fatores devem ser considerados: a provisão dos recursos materiais e humanos necessários, organização e sistematização de rotinas com procedimentos benéficos, prezando os princípios éticos e evitando-se o modelo intervencionista. $^{2}$

Existem duas possíveis vias de parto: parto cesáreo, somente realizado pelo médico obstetra em conjunto com sua equipe, por meio de uma conduta cirúrgica; e o parto normal simples em vértice que pode ser realizado tanto pelo médico quanto por um(a) enfermeiro(a) obstetra.

O parto normal é o mais indicado e seguro visto que o corpo feminino mantém uma preparação própria para a realização do mesmo, no entanto pode levar um período maior de trabalho de parto além de ser mais propenso à dor. $\mathrm{O}$ parto normal proporciona às mulheres força para lidar com a parturição, visando a dor como um aspecto intrínseco do ato de parir, transformando esse momento de sensação física e dolorosa em sentimento de amor, vivenciando o protagonismo feminino proporcionado pela maternidade. ${ }^{3}$

Os Centros de Parto Normal são estabelecimentos destinados ao atendimento à mulher no período gravídico puerperal, regulamentados na política de assistência ao parto pelo Ministério da Saúde e visam a melhoria da qualidade da assistência na gestação, parto e pós-parto, de modo huma- nista, bem como também reduzir a mortalidade materna e perinatal. ${ }^{5,6}$

Importante compreender o perfil epidemiológico de puérperas e recém-nascidos, pois é possível obter dados que contribuem para o planejamento de estratégias e de ações para progressos da assistência qualificada a essa clientela. Inúmeras variáveis interferem no processo saúde-doença, por isso o conhecimento da epidemiologia de uma população favorece um cuidado integral e eficaz em seu atendimento. ${ }^{7,8}$

Assim, o presente estudo tem por objetivo conhecer aspectos epidemiológicos das puérperas e de seus neonatos atendidos em um Serviço de Parto Normal de um município do interior da Bahia.

\section{Procedimentos METODOLÓGICOS}

\section{Delineamento e população alvo}

O presente estudo trata-se de uma pesquisa de abordagem quantitativa, com caráter descritivo, do tipo corte transversal, realizado no período de 1 de janeiro a 31 de dezembro de 2020. O estudo foi desenvolvido na Santa Casa de Misericórdia de Ruy Barbosa (BA), um hospital público, filantrópico, de médio porte, com 112 leitos, que é referência na região e atende usuários do Sistema Único de Saúde (SUS), sistema nacional de saúde pública brasileiro.

A referida instituição oferece assistência à saúde da mulher durante o processo de parturição, assistido por enfermeiros obstétricos e médicos, e, durante o período puerperal, no qual as puérperas permanecem internadas nos quartos de pré-parto, parto e pós-parto (PPP) e/ou enfermaria, em média, por 48 horas após o parto. 


\section{Local de realização do estudo}

O estudo foi desenvolvido na Santa Casa de Misericórdia de Ruy Barbosa (BA), um hospital público, filantrópico, de médio porte, que é referência na região e atende usuários do Sistema Único de Saúde (SUS), sistema nacional de saúde pública brasileiro.

O referido hospital está localizado no município de Ruy Barbosa, localizado no Piemonte da Chapada Diamantina, há aproximadamente $318 \mathrm{~km}$ da capital baiana, com área de $2.024 \mathrm{~km} 2$, com uma população estimada em 2020 de 30.857 habitantes (13,76 habitantes $/ \mathrm{km}^{2}$ ), renda mensal per capita de 1,5 salários e Índice de Desenvolvimento Humano (IDH) de 0,610 (Instituto Brasileiro de Geografia e Estatística - IBGE, 2020). ${ }^{9}$

\section{Casuística}

Participaram 276 puérperas e 276 neonatos, sendo que a amostra foi determinada por meio de procedimento amostral não probabilístico, do tipo "conveniência", definida de acordo com os seguintes critérios de inclusão: todas as mulheres que deram entrada na unidade hospitalar para atendimento obstétrico e que evoluíram em trabalho de parto, aquelas que tiveram RN nativivos com Índice de Apgar maior ou igual a 1 e gemelares no período da pesquisa. Os critérios de exclusão corresponderam às puérperas internadas na maternidade que estavam em isolamento no momento da coleta de dados, impossibilitadas de deambularem até a sala de entrevista, deficientes auditivas $\mathrm{e}$ as que foram submetidas à cesariana.

A pesquisa foi aprovada pelo Comitê de Ética em Pesquisa (CEP) da Faculdade Anísio Teixeira (FAT) de Feira de Santana (BA), parecer 1.078.653, seguindo os preceitos Éticos em Pesquisa com Seres Humanos, de acordo com a resolução do Conselho Nacional de Saúde/MS 466/2012. ${ }^{10}$

As participantes foram incluídas na pesquisa posteriormente à assinatura do Termo de Consentimento Livre e Esclarecido (TCLE) e o sigilo destas foi garantido por meio da substituição dos nomes por códigos alfanuméricos conforme ordem dos prontuários (PUERP 1, PUERP 2, etc.).

Os dados sociodemográficos e clínicos foram obtidos por meio do cartão da gestante, prontuário médico das mesmas e no caso de dados incompletos, os mesmos foram respondidos diretamente pela puérpera em quarto reservado da instituição, para garantir a privacidade das participantes, sem que houvesse a interferência de outros indivíduos, somente puérpera e pesquisador. Antes da entrevista foi apresentado o objetivo da pesquisa e informação sobre o pesquisador que iria conduzi-la.

\section{Variáveis sociodemográficas}

Informações referentes aos aspectos sociodemográficos, hábitos de vida e condições de saúde foram coletadas da avaliação dos dados descritos no prontuário e categorizadas para análise. As variáveis incluídas e suas respectivas categorizações foram: idade $(<16$ anos, 17 a 26 anos, 27 a 36 anos e $>37$ anos), cor da pele (branca ou outras), escolaridade ( 2 a 3 anos, 4 a 5 anos, $>6$ anos), tem companheiro (sim ou não), trabalho (sim ou não) e número de filhos (1, 2, 3, 4 ou mais).

\section{Variáveis clínicas}

Histórico materno e dos recém-nascidos foram coletados a partir da descrição das informações presentes nos prontuários. 
As variáveis do quadro clínico materno avaliadas foram: número de consultas de pré-natal $(<6$ ou $>6)$, uso do sulfato ferroso ( $\operatorname{sim}$ ou não), uso de drogas lícitas (sim ou não), comorbidades como hipertensão ( ou não) e diabetes ( $\operatorname{sim}$ ou não), histórico de aborto (sim ou não), episiotomia no parto atual (sim ou não), laceração (sim ou não) e grau da laceração no parto atual (1, 2 ou 3 ), profissional que assistiu o parto (médico e enfermeiro ou enfermeiro obstétrico) e utilização de métodos alternativos durante o trabalho de parto (bola suíça, banho morno, cavalinho, musicoterapia, massagem).

As variáveis do quadro clínico dos recém-nascidos foram: idade gestacional (pré-termo, a termo ou pós-termo), Apgar no $1^{\circ}$ minuto de vida (1 a 3, 4 a 6 e $>7$ ), Apgar no $5^{\circ}$ minuto de vida $(<5,6$ a $7,>8)$, o peso ao nascer $(<2500 \mathrm{~g},<2501 \mathrm{~g}$ e $>4000 \mathrm{~g})$, o sexo (masculino ou feminino) e o perímetro cefálico (inferior a $31,5 \mathrm{~cm}$, entre 32 e $36,8 \mathrm{~cm}$ ou superior a $37 \mathrm{~cm}$ ).

\section{Classificação dos neonatos quanto ao peso no nascimento e idade gestacional}

Para a classificação dos neonatos quanto ao peso ao nascimento, prematuridade e perímetro cefálico, foram utilizados como referência a definição da Organização Mundial da Saúde (OMS, 2002), ${ }^{8}$ que considera $\mathrm{RN}$ de baixo peso aqueles com menos de $2.500 \mathrm{~g}$, prematuros os nascidos com idade gestacional (IG) entre 20 e 36 semanas e 6 dias de gestação, a termo aqueles com IG entre 37 e 42 semanas, pós-termo aqueles nascidos após 42 semanas de gestação, e o perímetro cefálico quando entre $31,5 \mathrm{~cm}$ e $37 \mathrm{~cm}$, o considerado com tamanho normal.

\section{ANÁlise dos Dados}

As informações obtidas foram armazenadas no software aplicativo Microsoft Excel $^{\oplus}$ e a análise estatística descritiva foi realizada empregando-se o programa SPSS (Statistical Package for Social Science), versão 17.0 e o programa STATA (Software for Statistics and Data Science) versão 10.0 e versão 11.0, calculando-se as frequências absolutas e relativas para todas variáveis estudadas.

\section{Resultados}

Das 276 gestantes consultadas no período analisado, 60,5\% possuíam entre 17 e 26 anos de idade; $86,6 \%$ eram casadas ou mantinham união estável; 60,9\% declararam ser de cor parda; $90,2 \%$ relataram ter estudado por mais de 6 anos; $55,45 \%$ não trabalhavam e, a maioria $(43,5 \%)$ era primigesta, conforme observa-se na Tabela 1.

$\mathrm{Na}$ tabela 2 são apresentadas as características relacionadas à história materna e parto atual das puérperas do estudo. Observou-se que a maioria $(88,4 \%)$ realizou pelo menos de 6 consultas no pré-natal; $97,1 \%$ usaram sulfato ferroso na gestação; $94,2 \%$ relataram que não consumiram drogas lícitas durante a gestação; $85,1 \%$ não tinham hipertensão; $94,6 \%$ não tinham diabetes gestacional; e 91,3\% negaram histórico de aborto.

Quanto ao trabalho de parto atual, percebeu-se que 95,0\% das participantes do estudo não submeteram-se à episiotomia eem 45,7\% houve laceração, dentre essas, 25,4\% tiveram laceração grau 1, não requerendo reparo perineal (Tabela 2 ). Vale salientar que dentre as mulheres que tiveram episiotomia, $50,0 \%$ também apresentaram laceração de $2^{\circ}$ grau em virtude de tal procedimento. 
Tabela 1: Características epidemiológicas das puérperas atendidas no Serviço de Parto Normal em 2020 ( $n=276)$. Ruy Barbosa, Bahia, 2020.

\begin{tabular}{|c|c|c|}
\hline Variável & N (276) & $\%$ \\
\hline \multicolumn{3}{|l|}{ Idade da mãe } \\
\hline$<16$ anos & 17 & 6,2 \\
\hline 17 a 26 anos & 167 & 60,5 \\
\hline 27 a 36 anos & 83 & 30,0 \\
\hline$>37$ anos & 09 & 3,3 \\
\hline \multicolumn{3}{|l|}{ Cor (autorreferida) } \\
\hline Branca & 72 & 26,1 \\
\hline Parda & 168 & 60,9 \\
\hline Negra & 36 & 13,0 \\
\hline \multicolumn{3}{|l|}{$\begin{array}{l}\text { Escolaridade (anos de } \\
\text { estudo) }\end{array}$} \\
\hline $0-2$ anos & 06 & 2,2 \\
\hline $3-5$ anos & 21 & 7,6 \\
\hline$>6$ anos & 249 & 90,2 \\
\hline \multicolumn{3}{|l|}{ Tem companheiro } \\
\hline Sim & 239 & 86,6 \\
\hline Não & 37 & 15,4 \\
\hline \multicolumn{3}{|l|}{ Trabalho } \\
\hline Sim & 123 & 44,6 \\
\hline Não & 153 & 55,4 \\
\hline \multicolumn{3}{|l|}{$\begin{array}{l}\text { Número de filhos } \\
\text { (incluindo o recém- } \\
\text { nascido no dia da coleta } \\
\text { de dados) }\end{array}$} \\
\hline $\mathrm{Um}$ & 120 & 43,5 \\
\hline Dois & 76 & 27,5 \\
\hline Três & 36 & 13,0 \\
\hline Quatro ou mais & 44 & 16,0 \\
\hline
\end{tabular}

Fonte: Elaborada pelo responsável do estudo, Ruy Barbosa,Bahia, 2020.
Houve 14 partos em que se realizou episiotomia. Desse quantitativo, em 50\% a prática ocorreu pelo profissional médico, em dois partos a apresentação fetal estava pélvica e as parturientes estavam com IG de 41 semanas. Em 08 partos as mulheres eram primigestas, e, dentre essas, 02 com idade inferior a 17 anos e com 40 semanas de gestação.

O partejo foi conduzido, em sua maioria, pelos enfermeiros obstétricos $(94,6 \%)$ e com a utilização de manejos alternativos para alívio de dor e progresso do trabalho de parto, tais como deambulação (95,0\%), banho morno de aspersão (90,9\%), massagens $(89,5 \%)$, bola suíça $(84,0 \%)$ e cavalinho (44,6\%).

Tabela 2: Características relacionadas à história materna e parto atual das puérperas do estudo ( $\mathrm{n}=276)$. Ruy Barbosa, Bahia, 2020.

\begin{tabular}{|c|c|c|}
\hline Variável & $\begin{array}{c}\mathrm{N} \\
(276)\end{array}$ & $\%$ \\
\hline $\begin{array}{l}\text { Número de consultas de } \\
\text { pré-natal }^{*}\end{array}$ & & \\
\hline$<6$ & 31 & 11,3 \\
\hline$\geq 6$ & 244 & 88,7 \\
\hline
\end{tabular}

Uso de sulfato ferroso

$\begin{array}{lcc}\text { Sim } & 268 & 97,1 \\ \text { Não } & 08 & 2,9\end{array}$

Uso de drogas lícitas

Sim

$16 \quad 5,8$

Não

26094,2

Hipertensão

Sim

$41 \quad 14,9$

Não

$235 \quad 85,1$

Diabetes

Sim

$15 \quad 5,4$


continuação da Tabela 2

\begin{tabular}{|c|c|c|}
\hline Variável & $\begin{array}{c}\mathrm{N} \\
(276)\end{array}$ & $\%$ \\
\hline Não & 261 & 94,6 \\
\hline \multicolumn{3}{|l|}{ História de aborto } \\
\hline Sim & 24 & 8,7 \\
\hline Não & 252 & 91,3 \\
\hline \multicolumn{3}{|l|}{ Episiotomia } \\
\hline Sim & 14 & 5,0 \\
\hline Não & 262 & 95,0 \\
\hline \multicolumn{3}{|l|}{ Laceração } \\
\hline Sim & 126 & 45,7 \\
\hline Não & 150 & 54,3 \\
\hline \multicolumn{3}{|l|}{ Grau da laceração } \\
\hline 1 & 70 & 25,4 \\
\hline 2 & 55 & 20,0 \\
\hline 3 & 01 & 0,4 \\
\hline \multicolumn{3}{|l|}{ Parto assistido por } \\
\hline Médico e enfermeiro(a) & 15 & 5,4 \\
\hline Enfermeiro(a) obstétrico(a) & 261 & 94,6 \\
\hline \multicolumn{3}{|l|}{$\begin{array}{l}\text { Métodos alternativos para } \\
\text { alívio de dor }\end{array}$} \\
\hline Bola suíça & 232 & 84,0 \\
\hline Banho morno & 251 & 90,9 \\
\hline Cavalinho & 123 & 44,6 \\
\hline Musicoterapia & 22 & 8,0 \\
\hline Massagem & 247 & 89,5 \\
\hline Deambulação & 262 & 95,0 \\
\hline Agachamento & 241 & 87,3 \\
\hline Escada de Ling & 204 & 73,9 \\
\hline
\end{tabular}

*Total de mulheres que realizaram consulta de pré-natal $=275$.

Fonte: Elaborada pelo responsável do estudo, Ruy Barbosa,Bahia, 2020.

Houve 276 neonatos, sendo que desses, $50,4 \%$ eram do sexo masculino e $90,2 \%$ com peso adequado ao nascer. A Tabela 3 resume
Tabela 3: Características dos recém-nascidos avaliados no local e período do estudo $(n=276)$. Ruy Barbosa, Bahia, 2020.

\begin{tabular}{rcr}
\hline Variável & $\mathrm{N}$ & $\%$ \\
\hline Idade gestacional &
\end{tabular}

Idade gestacional

Pré-termo

$27 \quad 9,8$

A termo

$244 \quad 88,4$

Pós-termo

$05 \quad 1,9$

Apgar $1^{\circ}$ minuto

1 a 3

$01 \quad 0,4$

4 a 6

$10 \quad 3,6$

$>7$

265

96,0

Apgar $5^{\circ}$ minuto

$\begin{array}{lcc}<5 & 01 & 0,4 \\ 6 \text { a } 7 & 03 & 1,1 \\ >8 & 272 & 98,5\end{array}$

\section{Peso do Recém-nascido}

$\begin{array}{ccc}<2500 \mathrm{~g} & 13 & 4,7 \\ >2501 \mathrm{~g} & 249 & 90,2 \\ >4000 \mathrm{~g} & 14 & 5,1\end{array}$

\section{Sexo do Recém-nascido}

Masculino

13950,4

Feminino

$137 \quad 49,6$

Perímetro cefálico

Inferior a $31,5 \mathrm{~cm} \quad 20 \quad 7,2$

Entre 32 e $36,9 \mathrm{~cm} \quad 252 \quad 91,3$

\begin{tabular}{lll} 
Superior a $37 \mathrm{~cm}$ & 04 & 1,5 \\
\hline
\end{tabular}

Fonte: Elaborada pelo responsável do estudo, Ruy Barbosa, Bahia, 2020.

os indicadores coletados dos neonatos nascidos no local e período do estudo. Nota-se que RN a termo $(88,4 \%)$, com perímetro cefálico entre $31,5 \mathrm{~cm}$ e $37 \mathrm{~cm}(91,3 \%)$ e com Índice de Apgar maior que 7 no primeiro minuto $(96,0 \%)$ e maior que 8 no quinto minuto de vida $(98,5 \%)$ foram prevalentes entre os RN da amostra. 
Vale ressaltar que dos 13 neonatos com baixo peso ao nascer, 09 também eram prematuros e, dentre os neonatos com peso superior a $4.000 \mathrm{~g}$ nenhum era pós-termo.

\section{Discussão}

Foi possível conhecer os aspectos epidemiológicos das puérperas e de seus neonatos atendidos no Serviço de Parto Normal do município do local do estudo, tendo como principais achados: faixa etária das puérperas de 17 a 26 anos (60,5\%), mulheres casadas ou que mantinham uma união estável $(86,6 \%)$, primigestas $(43,5 \%)$; a maioria dos recém-nascidos era a termo $(88,4 \%)$ e teve índice de Apgar maior que 7 e 8 no primeiro $(96,0 \%)$ e no quinto $(98,5 \%)$ minuto de vida, respectivamente, e $4,8 \%$ nasceram com baixo peso.

Percebe-se prevalência de mães nas idades extremas, sendo 6,2\% mães adolescentes e 3,3\% mães com mais de 37 anos de idade, ambas com potencial de risco para a saúde materno-fetal., ${ }^{2,7}$ De acordo com a OMS, a gravidez na adolescência, aquela entre os 10 e 19 anos de idade, é considerada um problema de saúde pública, em virtude de ser caracterizada como fator de risco e vulnerabilidade, pois trata-se de um período de construção do indivíduo e inserção social. ${ }^{11-13}$ Dentre as complicações mais prevalentes na gestação de adolescentes, estão a pré-eclâmpsia, as infecções, o parto prematuro, as complicações no parto e puerpério e as perturbações emocionais, bem como as consequências associadas à decisão de abortar. ${ }^{14}$

Gestantes com idade superior a 35 anos apresentam uma incidência maior de complicações obstétricas, sendo a doença hipertensiva específica da gestação, diabetes ges- tacional, rotura prematura das membranas ovulares e hemorragias periparto as mais freqüentes. ${ }^{14}$ Além disso, os autores chamam a atenção para o fato de 6,0\% a 21,5\% das mulheres com idade avançada apresentarem trabalho de parto prematuro.

Verificou-se que a maioria das puérperas não tinha comorbidades. Desse modo, compreende-se que a condição de saúde da mulher está relacionada a desfechos desfavoráveis da gestação, tais como prematuridade e baixo peso. ${ }^{14,15}$

Quanto à situação conjugal, observa-se a prevalência de mulheres com companheiro, resultado similar ao de estudo que constatou que $75,7 \%$ e $83,6 \%$ das puérperas eram casadas ou tinham união estável. ${ }^{15,16}$

No presente estudo, 99,6\% da amostra realizaram consultas de pré-natal, sendo que a maioria participou de pelo menos 6 . Isso evidencia que as participantes seguiram as recomendações da OMS e do Ministério da Saúde em relação à importância de fazer as consultas do pré-natal. ${ }^{11,12} \mathrm{~A}$ justificativa para a não realização da consulta relatada pela única participante do estudo foi a situação de risco devido à pandemia da COVID-19. O pré-natal contribui para evitar desfechos desfavoráveis como o baixo peso do RN e a prematuridade. ${ }^{14,16}$

Acredita-se que a forma da organização dos serviços de saúde possa ser um fator de confusão da associação protetora de adequado pré-natal. Estudos verificaram que gestantes assistidas em menos de seis consultas de pré-natal tiveram aumento da chance para desfechos desfavoráveis tanto para o RN como para elas..$^{18-20}$

Durante a gestação, no presente estudo, observa-se um número reduzido de usuá- 
rias de drogas lícitas $(5,8 \%)$. Por meio das anotações no cartão das gestantes, percebe-se a eficácia das orientações e discussão realizadas nas consultas e em momentos de educação em saúde sobre os riscos que o tabagismo e o etilismo acarretam à mulher, tais como doenças cardiovasculares, placenta prévia, ruptura prematura das membranas, descolamento prematuro de placenta, câncer, distúrbios neurológicos e ganho de peso gestacional insuficiente; ao seu concepto e ao neonato: restrição do crescimento, aborto, baixo peso ao nascer, prematuridade, defeitos congênitos,síndrome alcoólica fetal, dentre outros. ${ }^{14,16,21}$

Quanto à realização de episiotomia, nesse estudo a taxa foi de 5,0\%, número aceitável conforme recomendação da OMS (entre $10 \%$ e 15\%). A episiotomia é considerada uma das maiores intervenções utilizadas em obstetrícia, sem evidências científicas de sua eficácia, e ainda hoje tem sido observada sua realização de forma rotineiras em muitas instituições. ${ }^{22-25}$

Todavia, existem algumas variáveis que podem influenciar na necessidade da realização da episiotomia, e que são apontadas pelo Ministério da Saúde como "considerar realização", tais como: macrossomia, variedade de posição, distocia de ombro e período expulsivo prolongado. ${ }^{24-26}$ Estudos salientam que a episiotomia prevalece em primíparas e sem consentimento ou conhecimento da parturiente. $^{25-28}$ No presente estudo isso foi verificado em parturientes primigestas e com idade inferior a 17 anos e todas as mulheres foram orientadas e deram consentimento para a realização desse procedimento.

Foi observado que a assistência durante o trabalho de parto, parto e puerpério ocor- reu em 94,6\% dos casos por enfermeiros obstétricos. Esses são considerados pela literatura os profissionais essenciais e responsáveis por acolher a mulher, assistir de modo humanizado, com qualidade e controle das atividades menos invasivas na assistência do período gravídico puerperal. ${ }^{28}$

Embora os "métodos não farmacológicos" ou "manejos alternativos para alívio da dor" não tirem a dor, promovem alívio e conforto. ${ }^{26,28}$ Consiste em atitudes e técnicas de promoção de relaxamento que tem como finalidades a diminuição de estresse e ansiedade da mulher durante o trabalho de parto; progresso da dilatação, além de evitar a medicalização durante esse período e proporcionar à mulher maior controle do seu parto. $^{15,28}$

Dentre os manejos alternativos para alívio da dor, nota-se que as parturientes realizaram exercícios em bola suíça, cavalinho e escada de Ling; foram orientadas/encaminhadas ao banho morno de aspersão, além disso, receberam massagens em regiões específicas durante o trabalho de parto. É papel fundamental do enfermeiro orientar à parturiente em relação à dor e desconforto no trabalho de partoe estimular o uso desses e outros métodos, como a deambulação e agachamentos. ${ }^{27,28}$

A maior parte dos bebês nasceu a termo $(88,4 \%)$, com perímetro cefálico dentro da normalidade $(91,3)$ e Índice de Apgar de primeiro e quinto minuto superior a 7 e 8 , respectivamente. A faixa de peso predominante foi de $2.500 \mathrm{~g}$ a $3.499 \mathrm{~g}$. Ressalta-se a significativa prevalência de baixo peso ao nascer $(4,8 \%)$ e prematuridade $(9,8 \%)$ entre os RN observados, valores que se apresentam parcialmente fora da média nacio- 
nal $(8,1 \%$ de baixo peso ao nascer e $6,5 \%$ de partos prematuros) apresentada pelos Indicadores e Dados Básicos (IDB) e Sistema de Informação sobre Nascidos Vivos (SINASC), respectivamente. ${ }^{12,13}$ Esses valores são preocupantes, pois os mesmos são considerados os fatores mais importantes na determinação da morbimortalidade neonatal assim como indicadores do estado de saúde das populações. ${ }^{14,15}$

Observou-se que as mães dos bebês com BPN realizaram menos consultas de pré-natal e usavam drogas lícitas. Estudos convergem com esse achado, evidenciando que o consumo de álcool e tabaco durante a gestação junto à redução da frequência às consultas de pré-natal caracterizam fatores de risco que promovem desfechos gestacionais negativos, o que pode ser justificado pela educação em saúde nessas consultas. ${ }^{16,18,29}$

Para que haja uma adequada assistência no Serviço do Parto Normal é imprescindível a integração e preparo da equipe multiprofissional no atendimento ao parto humanizado, com estrutura mínima para a gestante e seu RN. ${ }^{13}$ Assim, devem se fazer presentes médico obstetra, neonatologista, fisioterapeuta, dentre outros, para que junto aos enfermeiros obstetras consigam atuar de forma integral e qualificada. ${ }^{14,15}$

\section{Conclusão}

Os achados do estudo podem contribuir para o corpo de evidências em questão, pois elucidam semelhança com o perfil de puérperas apresentado na literatura, tais como conviver com companheiro, ter mais de 6 anos de estudo, realização de mais de 6 consultas de pré-natal, uso de sulfato ferroso na gestação e não ter comorbidades. Diante disso, devem ser observadas as limitações epidemiológicas do delineamento metodológico escolhido, por exemplo: vieses de memória devido ao seu caráter retrospectivo e possível constrangimento da puérpera durante a entrevista, além disso, os dados também foram obtidos por meio de uma revisão de prontuário que pode gerar algum viés de informação, como o confundimento e a falta de registro.

\section{REFERÊNCIAS}

1. Carvalho SS, Oliveira BR, Nascimento CSO, Gois CTS, Pinto IO. Percepção da equipe de enfermagem sobre a implantação do setor de acolhimento com classificação de risco às gestantes. Rev. Bras. Saúde Mater. Infant. 2018; 18(2):319-15

2. Reis JTS, Saraiva FO, Ferraresi MF, Vieira MAS. Perfil epidemiológico das parturientes atendidas em uma maternidade de alto risco de Goiânia-GO. estudos.2014;41(2):329-39

3. Zanatta E, Pereira CRR, Alves AP. Experiência da maternidade pela primeira vez: as mudanças vivenciadas no tornar-se mãe. Pesqui. prát. Psicossociais. 2018; 13(1):1-16

4. Silva ACL, Félix HCR, Ferreira MBG, Wysocki AD, Contim D, Ruiz MT. Preferência pelo tipo de parto, fatores associados à expectativa e satisfação com o parto.Rev. Eletr. Enf. 2017; 19:1-11

5. Riesco MLG, Oliveira SMJ, Bonadio IC, Schneck CA, Silva FMB, Diniz CSG, et al. Centros de Parto no Brasil: revisão da produção científica. Rev Esc Enferm USP. 2009; 43(supp 2): 1297-302

6. Brasil. Ministério da Saúde. Portaria $n^{\circ} 11$, de 7 de janeiro de 2015. Redefine as diretrizes para implantação e habilitação de centro de parto normal (CPN), no âmbito do sistema único de saúde (SUS), para o atendimento à mulher e ao recém-nascido no momento do parto e do nas- 
cimento, em conformidade com o componente PARTO E NASCIMENTO da Rede Cegonha, e dispõe sobre os respectivos incentivos financeiros de investimento, custeio e custeio mensal. Diário Oficial da República Federativa do Brasil, Brasília (DF), 2015.

7. Franciscatto LHG, Pasqua MD, Tolotti GK, Rossetto C, Argent C, Pinheiro JM. Delineamento do perfil epidemiológico de puérperas e recém-nascidos. Rev Enferm UFPE. 2014;8(5):1149-56

8. Lima JC, Oliveira GJ Júnior, Takano OA. Factors associated to fetal death in Cuiabá, Mato Grosso. Rev Bras Saude Mater Infant. 2016;16(3):353-61

9. Instituto Brasileiro de Geografia e Estatística. IBGE, 2020. Disponível em: URL <http://www. ibge.gov.br/>. Acesso em 12/11/2020.

10. Brasil. Ministério da Saúde. Conselho Nacional de Saúde. Resolução n 466, de 12 de dezembro de 2012. Diário Oficial da República Federativa do Brasil, Brasília (DF), 2012.

11. Organização Mundial da Saúde. Meeting of Advisory Group on Maternal Nutrition and Low Birthweight. Geneva: 2002:1-46.

12. Brasil. Ministério da Saúde. Secretaria de Atenção à Saúde. Departamento de Ações Programáticas Estratégicas. Atenção à Saúde do Recém-Nascido: Guia para os profissionais de saúde. 2. ed. Brasília: Ministério da Saúde; 2012.

13. BARROS F; MATIJASEVICH A.; SILVEIRA M. Consultoria: pesquisa para estimar a prevalência de nascimentos pré-termo no Brasil e explorar possíveis causas. Prematuridade e suas possíveis causas (Estudo). Unicef (BR); 2013.

14. Carvalho SS, Coelho JMF, Soares DAB, Mariola E. Fatores maternos para o nascimento de recém-nascidos com baixo peso e prematuros: estudo caso-controle. Ciência e Saúde. 2016; 9(2): 76-82

15. Barros MAR, Nicolau AIO. The pregnant woman's socioeconomic factors associated to the newborn infant's weight. J Nurs UFPE online. 2013;7(7):4769-74.

16. Carvalho SS, Coelho JMF. Perfil epidemiológico de puérperas de recém-nascidos com baixo peso e prematuros. Saúde Rev [Internet]. 2017 [cited 2020 Nov 12]; 17(45): 39-47. Disponível em: http://dx.doi.org/doi:10.15600/2238-1244/Sr.v17n45p39-4710

17. Allpas-Gómez HL, Raraz-Vidal J, Raraz-Vidal O. Factores associados al bajo peso al nacer em um hospital de Huánuco. Acta Méd Peru. 2014;31(2):79-83

18. Coelho JMF, Galvão CR, Rodrigues RM, Carvalho SS, Santos BM, Miranda SS, et al. Associação entre qualidade do pré-natal e baixo peso ao nascer em uma instituição hospitalar em Feira de Santana. Rev Epid Control Infec. 2018; 8(2): 129-35

19. Kehinde OA, Njokanma F, Olanrewaju DM. Parental socioeconomic status and birth weight distribuitionof Nigerian term newborn babies. Niger J Paed. 2013;40(3):299-302

20. Spiegler J, Jensen R, Segerer $H$, et al. Influence of smoking and alcohol during pregnancy on outcome of VLBW infants. Z Geburtshilfe Neonatol. 2013;217(6):215-9

21. Mizumoto BR, Moreira BM, Santoro-Lopes G, et al. Quality of antenatal care as a risk factor for early onset neonatal infections in Rio de Janeiro, Brazil. Braz J Infect Dis. 2015;19(3):272-7

22. Santos RCS, Santos RG. Fatores relacionados com a prática da episiotomia no Brasil: revisão de literatura. Estação Científica (UNIFAP). 2016; 6(2):43-52

23. Corrêa MD Junior, Passini R Júnior. Selective Episiotomy: Indications, Techinique, and Association with Severe Perineal Lacerations. Rev Bras Ginecol Obstet. 2016;38(6):301-7

24. Ballesteros-Meseguer C, Carrillo-García C, Meseguer-de-Pedro M, Canteras-Jordana M, Martínes-Roche ME. Episiotomy and its relationship to various clinical variables that influence its performance. Rev Latino-Am Enfermagem. 2016;24:e2793

25. Trinh AT, Roberts CL, Ampt AJ. Knowledge, attitude and experience of episiotomy use among obstetricians and midwives in Viet Nam. BMC Pregnancy Childbirth. 2015; 15:101 
26. Mellizo-Gaviria AM, López-Veloza LM, Montoya-Mora R, Ortiz-Martínez RA, Gil-Walteros GC. Frequency of episiotomy and complications in the obstetrics service of Hospital Universitario San José, Popayán (Colombia), 2016. Exploration of maternal and perinatal factors associated with its performance. RevColombObstetGinecol. 2018; 69(2): 88-97

27. Ballesteros-Meseguer C, Carrillo-García C, Meseguer-de-Pedro M, Canteras-Jordana M, Martínez-Roche ME. La episiotomía y surelacióncon distintas variables clínicas que influyen em surealización. Rev Lat Am Enfermagem. 2016;24:e2793

28. Silva DAO, Ramos MG, Jordão VRV, Silva RAR, Carvalho JBL, Costa MMN. Uso de métodos não farmacológicos para o alívio durante o trabalho de parto normal: revisão integrativa. Rev Enferm UFPE. 2013; 7:4161-70

29. Carvalho SS, Oliveira BR. Fatores preditivos para a ocorrência de baixo peso ao nascer e prematuridade: um estudo caso-controle. Rev Educ Saúde. 2019; 7 (1): 100-8

\section{DADOS DO AUTOR}

\section{Silas Santos Carvalho}

Mestre em saúde coletiva. Enfermeiro obstétrico da Santa Casa de Misericórdia, de Ruy Barbosa, Ruy Barbosa/BA - Brasil. ssc.academico@hotmail.com

Submetido em: 11-1-2021

Aceito em: 8-7-2021 